\title{
Neurological syndromes of brucellosis
}

\author{
M BAHEMUKA,* A RAHMAN SHEMENA, C P PANAYIOTOPOULOS, \\ A K AL-ASKA, TAHIR OBEID, A K DAIF
}

From the College of Medicine, Department of Medicine, Riyadh, Saudi Arabia

SUMMARY Eleven patients with brucellosis presented with neurological features closely simulating transient ischaemic attacks, cerebral infarction, acute confusional state, motor neuron disease, progressive multisystem degeneration, polyradiculoneuropathy, neuralgic amyotrophy, sciatica and cauda equina syndrome. Most patients improved quickly after adequate antibiotic treatment but chronic cases responded poorly. These protean neurological manifestations of brucellosis indicate that the underlying pathological mechanisms are diverse.

Brucellosis is a common infection in Saudi Arabia and still occurs in most developed countries. ${ }^{12}$ The nervous system may be affected diversely with complex symptomatology arising from the involvement of the meninges, cerebral vessels, central nervous system and cranial and peripheral nerves. ${ }^{2-5}$

The purpose of this report is to document our experience of 11 cases whose presentation mimicked several neurological syndromes and diseases, most of which improved with treatment. The cases with acute meningitis are not included as they have already been described elsewhere. ${ }^{6}$

\section{Patients and methods}

All patients suspected of brucellosis on the basis of their clinical features had blood taken for brucella culture and standard (Widal) agglutination tests for Brucella abortus and melitensis titres. For the primary culture, tryptic soy broth was used and if positive, the subculture was done on blood agar incubated in $10 \% \mathrm{CO}_{2}$ for at least 48 hours. The primary culture was usually kept for at least 6 weeks if the initial result was negative. The tests for antibiotic sensitivities were done by disc diffusion technique using isosensi test media. Those patients with neurological symptoms or signs indicating a mass lesion in the central nervous system had CT scan of the brain or myelography. Electromyography (EMG), nerve conduction and evoked response studies were done whenever indicated. Cerebrospinal fluid (CSF) was taken for cells, protein, glucose, culture and brucella titres in nine patients. Brucella species were identified on the basis of established micro-biological criteria but facilities for identification of individual species are not yet available.

* Dr Bahemuka died in an accident after acceptance of this paper.

Address for reprint requests: $\operatorname{Dr}$ A K Al-Aska MD, College of Medicine, Department of Medicine (38), Division of Neurology, PO Box 2925, Riyadh 11461, Saudi Arabia.

Received 22 September 1987 and in revised form 8 December 1987. Accepted 15 December 1987

\section{Case reports}

Case 1 A 26 year old right handed Sudanese was admitted with a day's history of headache, vomiting, drowsiness, confusion and fever. He had experienced intermittent bifrontal headache with no special features for 8 months. During this period he had recurrent fever, was anorexic and had lost weight. Four months prior to admission he had been having episodes of weakness in the right limbs associated with difficulties in choosing the correct words although he could understand what was said to him. Most of these episodes lasted about 10 minutes and none lasted more than an hour. He often drank unpasteurised goat and camel milk.

On examination he looked ill, was wasted and had a temperature of $39^{\circ} \mathrm{C}$. He was drowsy and confused but easily rousable. He had neck stiffness, positive Kernig's sign and mild left hemiparesis. Clinical and CSF findings (table 1) were compatible with tuberculosis and he was started on streptomycin, rifampicin, INH and ethambutol while awaiting further laboratory results including tests for brucellosis. The following day he was fully alert, orientated and apryexial. Brucella species were later isolated from the CSF and brucella agglutination titres were raised in both serum and CSF (Table 1). Detailed investigations for stroke risk factors such as echocardiography, electrocardiography, haemoglobin electrophoresis, serum lipid analysis and serological tests for collagen disorders were normal.

The antituberculosis drugs, except rifampicin, were discontinued and replaced by trimethoprim-sulfamethoxazole (TMP-SMZ). The patient continued to improve clinically and his antibody titres declined. He was discharged symptom free 5 weeks later. When reviewed 9 months after discharge he was well without any recurrence clinically and the brucella agglutination titres were $1 / 160$ for both abortus and melitensis respectively. However, minimal pyramidal tract signs (pathologically brisk tendon jerks) were still present in the left limbs.

Case 2 A 60 year old Saudi female was admitted with recurrent vertigo, nausea, vomiting, anorexia and weight loss for 3 months. The vertigo was worsened by exercise or change of position of the head. There was no history of headache, tinnitus, loss of hearing or visual disturbance. Six 
Table 1 Laboratory Investigations in 11 cases of neurobrucellosis

\begin{tabular}{|c|c|c|c|c|c|c|c|c|}
\hline \multirow{3}{*}{$\frac{\text { Case }}{1}$} & \multicolumn{2}{|c|}{ Serum Brucella Titres } & \multirow{3}{*}{$\begin{array}{l}\text { Blood culture } \\
\begin{array}{l}\text { Negative } \\
\text { (CSF positive) }\end{array}\end{array}$} & \multirow{2}{*}{\multicolumn{2}{|c|}{ Antibiotic sensitiveness }} & \multicolumn{3}{|c|}{ CSF examination } \\
\hline & Abortus & Melitensis & & & & Protein g/l & Glucose mmol/l & Cells \\
\hline & $\frac{1}{640}$ & $\frac{1}{160}$ & & $\begin{array}{l}\text { Tetracycline } \\
\text { Streptomycin } \\
\text { \& Rifampicin } \\
\text { TMP-SMZ }\end{array}$ & $\begin{array}{l}\text { Sensitive (S) } \\
\text { S } \\
\text { S } \\
\text { Resistant (R) }\end{array}$ & 1.4 & $1 \cdot 8$ & $\begin{array}{l}520 \\
95 \% \text { Lymphocyte }\end{array}$ \\
\hline 2 & $\frac{1}{1280}$ & $\frac{1}{1280}$ & Brucella species & $\begin{array}{l}\text { Tetracycline } \\
\text { Streptomycin } \\
\text { TMP-SMZ }\end{array}$ & $\begin{array}{l}\mathbf{S} \\
\mathbf{S} \\
\mathbf{R}\end{array}$ & 0.48 & $4 \cdot 1$ & 4 \\
\hline 3 & $\frac{1}{320}$ & $\frac{1}{320}$ & Brucella species & $\begin{array}{l}\text { Tetracycline } \\
\text { Streptomycin } \\
\text { Trimethoprim }\end{array}$ & $\begin{array}{l}\mathbf{S} \\
\mathbf{S} \\
\mathbf{R}\end{array}$ & $0 \cdot 24$ & $4 \cdot 2$ & 9 \\
\hline 4 & $\frac{1}{640}$ & $\frac{1}{640}$ & Negative & - & & 0.97 & $5 \cdot 1$ & $14095 \% \mathrm{~L}$ \\
\hline 5 & $\frac{1}{320}$ & $\frac{1}{320}$ & Negative & - & & $5 \cdot 9$ & 0.9 & $18090 \% \mathrm{~L}$ \\
\hline 6 & $\frac{1}{2560}$ & $\frac{1}{2560}$ & Brucella species & $\begin{array}{l}\text { Tetracycline } \\
\text { Streptomycin }\end{array}$ & $\begin{array}{l}\mathbf{S} \\
\mathbf{S}\end{array}$ & - & - & - \\
\hline 7 & $\frac{1}{1280}$ & $\frac{1}{1280}$ & Negative & - & & - & - & - \\
\hline 8 & $\frac{1}{2560}$ & $\frac{1}{2560}$ & Negative & - & & $6 \cdot 5$ & $4 \cdot 1$ & 225 mainly $\mathrm{L}$ \\
\hline 9 & $\frac{1}{1280}$ & $\frac{1}{1280}$ & Brucella species & $\begin{array}{l}\text { Tetracycline } \\
\text { Streptomycin } \\
\text { TMP-SMZ }\end{array}$ & $\begin{array}{l}\mathbf{S} \\
\mathbf{S} \\
\mathbf{R}\end{array}$ & 0.09 & $4 \cdot 4$ & 0 \\
\hline 10 & $\frac{1}{1280}$ & $\frac{1}{1280}$ & Brucella species & $\begin{array}{l}\text { Tetracycline } \\
\text { Streptomycin } \\
\text { TMP-SMZ }\end{array}$ & $\begin{array}{l}\mathbf{S} \\
\mathbf{S} \\
\mathbf{R}\end{array}$ & $1 \cdot 75$ & $3 \cdot 1$ & 6 \\
\hline 11 & $\frac{1}{1280}$ & $\frac{1}{1280}$ & Negative & - & & $5 \cdot 0$ & $1 \cdot 6$ & $29030 \% \mathrm{~L}$ \\
\hline
\end{tabular}

months prior to admission she had bouts of vertigo which lasted 2 weeks and improved.

On examination she looked ill, had a temperature of $38^{\circ} \mathrm{C}$ and a palpable spleen. The blood pressure was on several occasions $140 / 90 \mathrm{mmHg}$ lying down but $110 / 80 \mathrm{mmHg}$ standing up. This procedure also precipitated vertigo. She had trunkal ataxia but no other neurological signs. Urine culture grew Streptococcus faecalis for which she was treated with ampicillin $500 \mathrm{mg}$ six hourly and TMP-SMZ two tablets twice daily while awaiting the results of the tests for brucellosis. However, there was marked improvement on this regimen and at the time of discharge 3 weeks later she was free of symptoms. In the meantime investigations showed high brucella agglutination titres and brucella species were grown from blood culture (table 1). The neurological tests (table 2) were normal.

Six months later she had recurrence of her symptoms. This coincided with high Brucella abortus and melitensis titres of $1 / 320$ but she was subsequently lost to follow-up.

Case 3 A 60 year old Saudi female was admitted with a 2 week history of left sided weakness of gradual onset with sudden deterioration. There was no disturbance of consciousness and she was not known to be hypertensive or diabetic. She had had mild non-specific headache intermittently for a year and recurrent joint pains for a month prior to admission. She had been drinking raw goat and camel milk regularly for several months and several members of her family were already known to have brucellosis for which they were receiving treatment at this hospital.

On examination she had a temperature of $39.5^{\circ} \mathrm{C}$ and a palpable spleen. She was fully conscious and orientated.
There was a mild left hemiparesis with the face and arm more affected than the leg. The cardiovascular system including the blood pressure was normal. Serial CT brain scans with contrast were normal.

The diagnosis of brucellosis was confirmed by the finding of high brucella agglutination titres in serum and subsequent culture of brucella species from blood (Table 1) after which she responded well to a 3 week course of tetracycline and physiotherapy and was discharged home 2 months later. When seen a year later she was independent although still hemiparetic.

Case 4 A 40 year old Saudi was admitted with a week's history of generalised headache and loss of appetite. He became confused and was unable to recognise family members. He used to drink raw camel and sheep milk and had been treated for brucellosis until 2 months prior to admission. Neither the antibiotics nor the duration of treatment were known.

On examination he was restless, confused, perseverating and could not recognise friends or close members of the family. His temperature was $38 \cdot 5^{\circ} \mathrm{C}$. He had no neck stiffness or any other neurological signs. Investigations confirmed the diagnosis of brucellosis (tables 1 and 2). His mental state improved within 2 days of treatment with doxycycline and was normal a week later.

Case 5 A 19 year old Saudi male complained of progressive weakness of all limbs, loss of balance, vertigo, blurring of vision, double vision and headache for 17 months. He gradually became deaf during the 8 months prior to admission. There was no history of raw milk ingestion.

On examination he had bilateral sensorineural deafness, 
Table 2 Presentation and neurological investigations of 11 cases of brucellosis

\begin{tabular}{|c|c|c|c|}
\hline Case & Type of presentation & Investigation & Result of test \\
\hline 1 & $\begin{array}{l}\text { Transient ischaemic attacks in the } \\
\text { anterior circulation and alternating } \\
\text { hemiparesis }\end{array}$ & CT brain scan & Normal \\
\hline 2 & $\begin{array}{l}\text { Vertigo, trunkal ataxia and postural } \\
\text { hypotension }\end{array}$ & $\begin{array}{l}\text { Audiometry, brainstem evoked } \\
\text { potentials, CT brain scan }\end{array}$ & Normal \\
\hline 3 & $\begin{array}{l}\text { Cerebrovascular accident } \\
\text { (middle cerebral artery territory) }\end{array}$ & $\begin{array}{l}\text { CT brain scan } \\
\text { EEG }\end{array}$ & $\begin{array}{l}\text { Normal } \\
\text { Slow waves in right parietal area }\end{array}$ \\
\hline 4 & Acute confusional state & CT brain scan & Normal \\
\hline 5 & $\begin{array}{l}\text { Polyradiculoneuropathy, } \\
\text { trunkal ataxia, extensor plantar } \\
\text { responses and neurogenic deafness }\end{array}$ & $\begin{array}{l}\text { Nerve conduction studies } \\
\text { Visual evoked potentials } \\
\text { Auditory brainstem evoked potentials }\end{array}$ & $\begin{array}{l}\text { Sensorimotor peripheral neuropathy } \\
\text { Delayed latencies bilaterally } \\
\text { None obtained }\end{array}$ \\
\hline 6 & $\begin{array}{l}\text { Motor neuron disease } \\
\text { (Amyotrophic lateral sclerosis) }\end{array}$ & $\begin{array}{l}\text { Nerve conduction studies } \\
\text { EMG of upper and lower limb muscles }\end{array}$ & $\begin{array}{l}\text { Normal } \\
\text { Fasciculation potentials with long } \\
\text { duration }\end{array}$ \\
\hline 7 & Unilateral brachial radiculopathy & $\begin{array}{l}\text { Motor nerve conduction velocities } \\
\text { EMG of the right deltoid, biceps and } \\
\text { brachioradialis }\end{array}$ & $\begin{array}{l}\text { Normal } \\
\text { No resting activity, polyphasic } \\
\text { potentials }\end{array}$ \\
\hline 8 & $\begin{array}{l}\text { Guillain Barré-like syndrome } \\
\text { (Symmetrical polyradiculopathy } \\
\text { with no sensory loss) }\end{array}$ & $\begin{array}{l}\text { Nerve conduction studies } \\
\text { EMG }\end{array}$ & $\begin{array}{l}\text { Normal } \\
\text { Denervation }\end{array}$ \\
\hline 9 & $\begin{array}{l}\text { Acute urinary retention, sciatica } \\
\text { and perianal hypoalgesia }\end{array}$ & $\begin{array}{l}\text { Lumbosacral radiography } \\
\text { Myelogram }\end{array}$ & $\begin{array}{l}\text { Normal } \\
\text { Normal }\end{array}$ \\
\hline 10 & Cauda equina syndrome & $\begin{array}{l}\text { EMG of tibialis anterior, } \\
\text { gastrocnemius and hamstrings } \\
\text { Nerve conduction studies } \\
\text { Myelography }\end{array}$ & $\begin{array}{l}\text { Denervation } \\
\text { Normal } \\
\text { Complete block at } \mathrm{L} 4 / 5\end{array}$ \\
\hline 11 & $\begin{array}{l}\text { Lateral lumbar disc prolapse with } \\
\text { sphincter dysfunction and myelopathy }\end{array}$ & Myelography & Obliteration of the L4 nerve root \\
\hline
\end{tabular}

flaccid areflexic quadriparesis with bilateral extensor plantar responses and ataxic gait out of proportion to the weakness. Both proximal and distal musculature were equally affected. Sensation was normal.

The EMG and the nerve conduction studies were consistent with mild sensorimotor polyradiculoneuropathy. Serological tests and CSF findings were compatible with brucellosis (table 1). After 2 months treatment with vibramycin, TMP-SMZ and rifampicin he was ataxic but able to walk independently. There was no recovery of hearing and the deep tendon jerks and plantar responses remained unchanged.

Case 6 A 60 year old Saudi male presented with a 6 month history of intermittent fever, general malaise, wasting and weakness of all his limbs and difficulty in walking.

On examination he was thin, had generalised muscle wasting and weakness which was greater in the lower limbs and brisk tendon jerks. The laboratory investigations, compatible with brucellosis are shown in table 1 . The nerve conduction studies and the EMG indicated diffuse motor neuron involvement. He responded well to streptomycin and doxycycline in standard doses for 10 days and 3 weeks respectively during which time he walked independently.

Case 7 A 60 year old Saudi male was seen in the medical out-patients with a 3 month history of general body aches, joint pains, low backache and intermittent fever. He regularly drank raw camel milk.

On examination he looked well, was apyrexial and had no abnormal physical signs. Brucella agglutination titres were raised (table 1). He was put on a course of TMP-SMZ two tablets twice daily but 2 weeks later he was admitted because of weakness of the right arm and increased pain in the right shoulder of 3 days duration.

Examination confirmed profound weakness without wasting or fasciculations of the right deltoid, biceps, brachioradialis and, to a lesser extent, the triceps. Passive movements of the right shoulder joint were normal. The shoulder abduction and elbow flexion were MRC grade 0 and 2 respectively. Right biceps and brachioradialis jerks were absent and triceps jerk was diminished.

TMP-SMZ was stopped and streptomycin $750 \mathrm{mg}$ intramuscularly once daily and tetracycline $500 \mathrm{mg}$ orally four times a day were started. The following day his shoulder pain was much less but he was still unable to move it voluntarily. However, after 5 days treatment he started to move his shoulder and by the end of 2 weeks he was generally much better and could move his right arm easily at all joints. At the time of discharge 18 days later he was symptom free, but the biceps and brachioradialis tendon jerks were still absent and remained so for the next 3 months. Right shoulder abduction, elbow flexion and extension improved to MRC grade 4, 6 weeks after admission.

Case 8 A 30 year old Saudi female complained of generalised weakness, fatiguability, intermittent headache, blurring of vision, vomiting and low grade fever for 4 months. Her symptoms had deteriorated and she was unable to walk in spite of being on antibiotics for a month prior to admission. There was no history of raw milk ingestion and she had not been in contact with farm animals. She had aborted at 5 weeks, 8 months previously.

On examination she was apyrexial. There was symmetrical mainly proximal flaccid areflexic quadriparesis. The lower limbs were more affected. Serum and CSF brucella agglutinin titres were raised and the CSF showed a high lymphocyte count and markedly raised protein content (table 1). The EMG showed denervation and the nerve conduction studies were normal. Sural nerve histology was normal. She has slowly improved on treatment with rifampicin and doxycycline and can now, 6 weeks later, walk with the aid of a Zimmer frame. 
Case 9 A 20 year old Saudi male who had been regularly consuming unpasteurised milk was admitted complaining of low back pain radiating to the lateral aspect of the right thigh for 5 months. The pain started suddenly and was aggravated by carrying heavy objects, coughing, sneezing and bowel movement. The day after the onset he could only walk with the aid of a stick. By that time the pain was radiating to both thighs. Three weeks later he experienced dribbling of urine and perianal paraesthesiae. Four days prior to admission he was unable to walk or pass urine. There were no systemic symptoms including fever. The systemic examination was normal but his temperature was $37.9^{\circ} \mathrm{C}$.

Straight leg raising was limited to $50^{\circ}$ on the right and to only $30^{\circ}$ on the left. Spinal flexion was also limited. The power and reflexes were normal. He had hypoaesthesiae in the sacral segments particularly perianally but the anal sphincter tone was normal. Myelography was normal. Brucella agglutination titres were high (table 1). His symptoms resolved within the first week of treatment with doxycycline $100 \mathrm{mg}$ twice daily. He started to walk on the third day of treatment and his urinary symptoms resolved. On discharge 3 weeks later he was free of any symptoms and remained so in the 3 months of follow-up.

Case 10 A 31 year old male Egyptian was admitted with a history of low backache for 2 years and gradual weakness of both legs. Examination showed limitation of spinal flexion and straight leg raising bilaterally. There was marked wasting and weakness of the anterior tibial and the calf muscles bilaterally. The knee jerks were preserved but the ankle jerks were absent. The plantar responses were flexor and there was no sensory loss.

Myelography demonstrated complete block at L4/5. Laminectomy showed a degenerative infected disc which on histology was surrounded with purulent exudate compatible with pyogenic infection but the gram and Ziehl-Neelsen stains and cultures were negative. Investigations confirmed brucellosis (table 1) for which he was treated successfully with doxycycline.

Case 11 A 30 year old Syrian male was admitted with a 10 day history of low back pain radiating to both thighs posteriorly and worse on sneezing or coughing. For 2 days prior to admission he was unable to walk or stand and had hesitancy of micturition.

On examination he had marked bilateral weakness of foot dorsiflexion and mild weakness of plantar flexion. The knee and ankle jerks were brisk. The plantar responses were flexor. There was hypoalgesia in the S3-S5 dermatomes bilaterally. The investigations summarised in tables 1 and 2 indicated brucellosis affecting lumbar discs. The patient improved on a course of TMP-SMZ and rifampicin.

\section{Discussion}

The diagnosis of brucellosis with involvement of the nervous system was based on symptoms and signs of neurological disease, raised serum brucella titres (greater than 1/160) with or without positive blood culture and subsequent clinical remission or improvement with antibiotic therapy. The serological criterion was based on a previous study from this centre of culture positive brucellosis which showed that four out of 30 patients had antibody titres of $1 / 320$ for Brucella melitensis and three others had titres of $1 / 160$ or less for Brucella abortus. ${ }^{7}$ The rapid improvement that occurred in some of our patients has also been observed in other patients elsewhere. ${ }^{5}$ It is worth noting that five of our patients (cases 2, 5, 8, 10 and 11) gave no history of exposure such as by raw milk ingestion or contact with farm animals which suggests a possibility of uncommon sources of infection.

The favourable clinical response to TMP-SMZ and ampicillin by case 2 , although the sensitivity studies showed the isolates to be resistant to this drug, suggests that ampicillin which this patient was taking may also be effective in brucellosis. An alternative explanation is that in vitro sensitivity studies of brucella organisms are a poor guide to the efficacy of co-trimoxazole (TMP-SMZ) in the treatment of brucellosis.

Transient ischaemic attacks which were the presenting symptoms in case 1 and probably case 2 and occlusive vascular disease (case 3 ) are well documented complications of brucellosis. ${ }^{2}$ However, alternating hemiparesis as occurred in case 1 seems to be rare. The clinical features of case 3 could be attributable to an occlusive vascular lesion in the middle cerebral artery territory but a focal inflammatory or demyelinating lesion could also account for this. ${ }^{3}$

Psychiatric manifestations of brucellosis are well known but acute confusional state like that of case 4 is rarely reported ${ }^{8}$ (quoted by $\mathrm{Sahs}^{2}$ ). It has been proposed that the pathological basis of this is multifactorial. Neuronal necrosis, chronic vasculitis and multiple non-caseating small granulomata have all been proposed. ${ }^{9}$

Case 5 with a chronic progressive course showed cranial nerve involvement, polyradiculoneuropathy and pyramidal and cerebellar signs. This combination of clinical features in absence of systemic symptoms closely mimicked a multisystem degenerative disorder of the nervous system.

Although cases 4 and 5 had relatively low titres of $1 / 640$ and $1 / 320$ respectively, this does not argue against the diagnosis of brucellosis because experience from this centre ${ }^{7}$ and Kuwait ${ }^{10}$ shows that patients with culture-proven brucellosis may have low serum titres, sometimes as low as $1 / 80$. These two patients had clinical picture and CSF findings (table 1) compatible with bacterial meningitis and responded favourably to treatment of brucellosis. Case 4 had a definite history of exposure and improved dramatically on doxycycline alone. Case 5 had polyradiculoneuropathy which is rare in any other 
type of granulomatous bacterial meningitis but is commonly seen in brucellosis in this region. ${ }^{4510}$

Motor neuron disease was the initial diagnosis of case 6. This form of presentation of brucellosis has not been previously described although anterior horn cell degeneration has been documented ${ }^{3}$ and brucellosis simulating acute poliomyelitis has been reported. ${ }^{11}$

The last five cases illustrate root involvement which may be localised or diffuse. The former may be due to a compressive lesion ${ }^{12}$ but a non-compressive process like demyelination of the nerve roots has also been suggested. ${ }^{23}$ The neurological features of cases 10 and 11 were due to infected prolapsed discs while case 9 most probably suffered from localised noncompressive lumbosacral radiculopathy with the brunt of the lesion affecting the sensory roots. Radiculopathy with negative myelogram commonly affects the lower limbs ${ }^{5}$ but unilateral acute brachioradiculopathy which may mimic brachial neuralgic amyotrophy (case 7) is rare. Both of these types of presentation with considerable overlap may be difficult to distinguish clinically and therefore myelography or other appropriate radiographic studies are necessary.

Brucellosis may also affect multiple nerve roots in upper and lower limbs thus simulating Guillain-Barré syndrome ${ }^{4} 5$ (case 8 ) or there may be other additional features such as central nervous sytem signs and cranial neuropathy (case 5).

The presenting features of cases 2 and 11 included postural hypotension and urinary retention respectively suggesting involvement of the autonomic nervous system which has been documented in this infection ${ }^{13}$ (quoted by $\mathrm{Sahs}^{2}$ ). However, transient lower urinary tract obstruction which has also been reported in brucellosis ${ }^{914}$ may account for the last patient's urinary symptoms.

Case 10 had clinical and EMG features of cauda equina syndrome which was caused by an infected central lumbar disc prolapse. The presence of brisk knee and ankle jerks in case 11 suggested that apart from radiculopathy caused by infected $L 4 / 5$ disc there was also minimal myelopathy.

Brucella species isolated from blood cultures in five cases and the CSF but not in blood in one (table 1) were resistant to cotrimoxazole in four and sulphonamide and trimethoprim in one case each. This indicates that TMP-SMZ should be used with other drugs like tetracycline and streptomycin which may be superior in the treatment of neurobrucellosis. Chronic cases particularly those with multisystem involvement may not respond well to treatment (case 5 ) but quick recovery is to be expected in acute cases. ${ }^{5}$ We suggest that rifampicin, a potent anti-tuberculosis drug, should be reserved for resistant cases only, par- ticularly in countries where tuberculosis is still common.

The protean manifestations of brucellosis of the nervous system suggest that there is more than one pathological mechanism involved. The diagnosis of brucellosis in patients presenting with neurological features may be missed or delayed as this infection can imitate various diseases or syndromes. It is therefore recommended that even without a definite history of exposure, the presence of an obscure or unexplained neurological deficit should alert the clinican to the possibility of brucellosis in countries where this infection is endemic.

\section{References}

1 Madkour MM, Abdel Rahman E, Mohamed E, Samad Talukder MA, Kudwah Aida JN. Brucellosis in Saudi Arabia. Saudi Med J 1985;6:324-32.

2 Sahs AL. Brucellosis (Malta fever, undulant fever). In: Vinken PJ, Bruyn GW, eds. Handbook of Clinical Neurology, Vol 33. Amsterdam: North Holland, 1978:305-26.

3 Fincham RW, Sahs AL, Joynt RJ. Protean manifestations of nervous system brucellosis: case histories of a wide variety of clinical forms. JAMA 1963; 184:269-76.

4 Bashir R, Al-Kawi MZ, Harder EJ, Jenkins J. Nervous system brucellosis: diagnosis and treatment. Neuro$\log y$ 1985;35:1576-81.

5 Shakir RA, Al-Din ASN, Araj GF, Lulu AR, Mousa AR, Saadah MA. Clinical categories of neurobrucellosis: a report of 19 cases. Brain 1987;110: 213-23.

6 Al-Orainey I, Laajam MA, Al-Aska AK, Rajapakse C. Brucella meningitis. J Infect 1987;14:141-5.

7 Kambal AM, Mahgoub ES, Jamjoom GA, Chowdhury MNH. Brucellosis in Riyadh, Saudi Arabia: a microbiological and clinical study. Trans $R$ Soc Trop Med 1983;77:820-24.

8 Spink WW, Hall WH. Encephalomeningitis due to brucella abortus. Trans Am Clin Climatol Assoc 1949;61:121-40.

9 Young EJ. Human brucellosis. Rev Infect Dis 1983; 5:821-42.

10 Mousa ARM, Koshy TS, Araj GF, et al. Brucella meningitis: Presentation, diagnosis and treatment: a prospective study of ten cases. $Q J$ Med 1986; New series 60:873-80.

11 Debono JE. Brucellosis simulating acute anterior poliomyelitis. Lancet 1964;i:1132-3.

12 Aguilar JA, Elvidge AR. Intervertebral disc disease caused by the brucella organism. $J$ Neurosurg 1961;18:27-33.

13 Roger H, Poursines Y. Les meningo-neurobrucelloses. Paris: Masson, 1938:248.

14 Boyd ML. Human prostatic infection with brucella abortus. J Urol 1938;39:717-21. 